COMMUNICATIONS IN

ANALYSIS AND GEOMETRY

Volume 12, Number 4, 837-852, 2004

\title{
A New Parabolic Flow in Kähler Manifolds
}

\author{
X. X. CHEN
}

\section{Introduction.}

This is a follow-up work of my earlier paper [8]. In [8], we study the lower bound of the K energy on the Kähler manifold when the first Chern class is negative. This is an important problem in Kähler geometry since the existence of lower bound of the K energy is the pre-condition for the existence of constant scalar curvature metric problem (cf. [2] and [9]). According to a decomposition formula in $[8]^{2}$, the problem is reduced to the problem of solving the existence of critical metrics of a new functional $J$ introduced both in our paper [8] and Donaldson's work [11]. For convenience, we include its definition below. The existence problem is completed solved in Kähler surface. However, the existence problem in general dimension is still open. In this paper, we try to understand the general existence problem in Kähler manifold via flow method. Let $\left(V^{n}, \omega_{0}\right)$ be a Kähler manifold and $\omega_{0}$ be any Kähler form in $V$. Consider the space of Kähler potentials

$$
\mathcal{H}=\left\{\varphi, \omega_{\varphi}=\omega_{0}+i \partial \bar{\partial} \varphi>0, \text { on } V\right\} .
$$

For any fixed positive $(1,1)$ form $\chi$, one introduces a new functional $J$ with respect to this form $\chi$. Denote $g=\sum_{\alpha, \bar{\beta}=1}^{n} g_{\alpha \bar{\beta}} d z_{\alpha} d \overline{z_{\beta}}$ is the Kähler metric corresponds to the Kähler form $\omega$.

Definition 1.1. Suppose $\chi$ is a closed $(1,1)$ form, then for any $\varphi(t) \in \mathcal{H}, J$ is defined through its derivative:

$$
\frac{d J}{d t}=\int_{V} \frac{\partial \varphi}{\partial t} \chi \wedge \frac{\omega_{\varphi}^{n-1}}{(n-1) !} .
$$

It is straightforward to show that this is well defined.

\footnotetext{
${ }^{1}$ This paper was accepted in 2000 ; but publication has been delayed.

${ }^{2}$ An explicit formula for the K energy was given first by Tian [23] earlier.
} 
Remark 1.2. This definition is given by [11]. In [8], we assume $\chi$ is a Ricci form, but not necessary positive.

In [11], Donaldson outlined the significance of this functional in its own right: a) $J$ is a convex functional in the space of Kähler potentials (See proposition 1 below); b) $J$ is a moment map from the space of Kähler potentials to the dual space of the Lie algebra of some sympletic automorphism group. In this paper, we will continue to study the existence problem of the critical point of $J$. In particular, we concentrate in the gradient flow of this functional.

The Euler equation for $J$ is:

$$
\sum_{\alpha, \beta=1}^{n} g^{\alpha \bar{\beta}} \chi_{\alpha \bar{\beta}}=\operatorname{tr}_{g} \chi=c
$$

where

$$
\chi=\sum_{\alpha, \beta=1}^{n} \chi_{\alpha \bar{\beta}} d z_{\alpha} d \overline{z_{\beta}} .
$$

Here $g$ is the critical metric and $c$ is a constant depending only on Kähler class of $[\chi]$ and $\left[\omega_{0}\right]$ :

$$
c=\frac{\int_{V} \chi \wedge \frac{1}{(n-1) !} \omega^{(n-1)}}{\int_{V} \frac{1}{n !} \omega^{n}}=\frac{n[\chi] \cdot\left[\omega_{0}\right]^{n-1}}{\left[\omega_{0}\right]^{n}} .
$$

Consider the parabolic equation

$$
\frac{\partial \varphi}{\partial t}=c-\sum_{\alpha, \beta=1}^{n} g^{\alpha \bar{\beta}}(\varphi) \chi_{\alpha \bar{\beta}} .
$$

Here

$$
g_{\alpha \bar{\beta}}(\varphi)=g_{0 \alpha \bar{\beta}}+\frac{\partial^{2} \varphi}{\partial z^{\alpha} \bar{\partial} z^{\bar{\beta}}} .
$$

Similar to the case of Calabi flow [4], the main result of this paper is

Theorem 1.3. The following statements are true:

1. This gradient flow of $J$ always exists for all time for any smooth initial data. Moreover, the length of any smooth curve and the distance between any two metrics decreases under this flow. 
2. If the bisectional curvature of $\chi$ is semi-positive, then the gradient flow exists for all time and converges to a smooth critical metric.

From equation (1), it is easy to see that a necessary condition for a solution to exist is (also see [11])

$$
c \cdot \omega_{g}-\chi>0,
$$

where $\omega_{g}$ is the Kähler form associated to $g$. In other words, there exists at least one Kähler form $\omega$ in the Kähler class of $\left[\omega_{0}\right]$ such that the following holds:

$$
c \cdot \omega-\chi>0 .
$$

Conjecture 1.4. (Donaldson [11]) If the aforementioned necessary condition is satisfied, then there exists a critical point for $J=J_{\chi}$ in that Kähler class.

Historic remarks: Using heat flow method to study the nonlinear PDE is a well known method in differential geometry. In recent years, it has been the source of active researches since the famous work of Eells J. and Sampson [13]. Interested readers are refereed to important work by Hamilton R. [16], Huisken G. [18][17] and a survey paper by Cao H.D. and Chow B.[7] and the references therein.

Acknowledgement The author would like to thank Professor Donaldson for his encouragement in studying the critical points for the functional $J$, which began in the spring of 1999 when the author visited him. The author also would like to thank Professor Huisken G. for many interesting discussion about heat flow and Hamilton's maximal principal for tensors, the later play a key role in proving Theorem 1.2. Also, the author would like to thank Schoen R. Simon L., Calabi E. and Tian G. for their interests in this work. The author is very grateful to the referee for pointing out a number of errors in the eariler version of this paper.

\section{Summary of recent developments in the Riemannian metric in space of Kähler potentials.}

Let $\left(V, \omega_{0}\right)$ be a Kähler manifold. Mabuchi ([19]) in 1987 defined a Riemannian metric on the space of Kähler metrics, under which it becomes (formally) a non-positive curved infinite dimensional symmetric space. Apparently unaware of Mabuchi's work, Semmes [21] and Donaldson [12] rediscover this same metric again from different angles. For any vector $\psi$ in 
the tangential space $T_{\varphi} \mathcal{H}$, we define the length of this vector as

$$
\|\psi\|_{\varphi}^{2}=\int_{V} \psi^{2} d \mu_{\varphi}
$$

The geodesic equation is

$$
\varphi(t)^{\prime \prime}-\frac{1}{2}\left|\nabla \varphi^{\prime}(t)\right|_{\varphi(t)}^{2}=0,
$$

where the derivative and norm in the 2 nd term of the left hand side are taken with respect to the metric $\omega_{\varphi(t)}$.

This geodesic equation shows us how to define a connection on the tangent bundle of $\mathcal{H}$. If $\phi(t)$ is any path in $\mathcal{H}$ and $\psi(t)$ is a field of tangent vectors along the path (that is, a function on $V \times[0,1]$ ), we define the covariant derivative along the path to be

$$
D_{t} \psi=\frac{\partial \psi}{\partial t}-\frac{1}{2}\left(\nabla \psi, \nabla \phi^{\prime}\right)_{\phi}
$$

This connection is torsion-free because in the canonical "co-ordinate chart", which represents $\mathcal{H}$ as an open subset of $C^{\infty}(V)$. The main theorem formally proved in [19] (and later reproved in [21] and [12]) is:

Theorem A The Riemannian manifold $\mathcal{H}$ is an infinite dimensional symmetric space; it admits a Levi-Civita connection whose curvature is covariant constant. At a point $\phi \in \mathcal{H}$ the curvature is given by

$$
R_{\phi}\left(\delta_{1} \phi, \delta_{2} \phi\right) \delta_{3} \phi=-\frac{1}{4}\left\{\left\{\delta_{1} \phi, \delta_{2} \phi\right\}_{\phi}, \delta_{3} \phi\right\}_{\phi},
$$

where $\{,\}_{\phi}$ is the Poisson bracket on $C^{\infty}(V)$ of the symplectic form $\omega_{\phi}$; and $\delta_{1} \phi, \delta_{2} \phi \in T_{\phi} \mathcal{H}$. Then the sectional curvature is non-positive, given by

$$
K_{\phi}\left(\delta_{1} \phi, \delta_{2} \phi\right)=-\frac{1}{4}\left\|\left\{\delta_{1} \phi, \delta_{2} \phi\right\}_{\phi}\right\|_{\phi}^{2} .
$$

We will skip the proof here. Interested readers are referred to paper of Mabuchi [19] or [21] and [12] for the proof.

This subject has been quiet since the early pioneer work of Mabuchi (1987) and Semmes(1991). The real breakthrough came in the beautiful paper by Donaldson [12] in 1996. In [12], he outlines the connection between this Riemannian metric in the infinite dimensional space $\mathcal{H}$ and the traditional Kähler geometry through a series important conjectures and theorems. In 1997, following his program, the author proves some of his conjecture:

Theorem B [9] The following statements are true: 
1. The space of Kähler potentials $\mathcal{H}$ is convex by $C^{1,1}$ geodesics. More specifically, if $\varphi_{0}, \varphi_{1} \in \mathcal{H}$ and $\varphi(t)(0 \leq t \leq 1)$ is a geodesic connecting these two points in $\mathcal{H}$, then the mixed convariant derivative of $\varphi(t)$ is uniformly bounded from above.

2. $\mathcal{H}$ is a metric space. In other words, the infimum of the lengths of all possible curves between any two points in $\mathcal{H}$ is strictly positive.

In [4], E. Calabi and the author proved the following:

Theorem $\mathbf{C}[4]$ The following statements are true:

1. $\mathcal{H}$ is a non-positive curved space in the sense of Alenxandrov.

2. The length of any curve in $\mathcal{H}$ is decreased under the Calabi flow unless it is represented by a holomorphic transformation. The distance in $\mathcal{H}$ is also decreaseing under various geometric condition.

\section{General preparation.}

In this section, we assume $\chi>0$ is a Kähler form and $J$ is defined with respect to this positive form $\chi$. Then

Proposition 3.1. $J$ is a strictly convex functional on any $C^{1,1}$ geodesic. In particular, $J$ has at most one critical point in $\mathcal{H}_{0}$.

Remark 3.2. This proposition was pointed to the author by Donladson in spring 1999 when the author visited him. The proof here is somewhat different than his original proof.

Proof. Suppose $\varphi(t)$ is a $C^{1.1}$ geodesic. In other words, $\varphi(t)$ is a weak limit of the following continuous equation as $\epsilon \rightarrow 0$ :

$$
\left(\frac{\partial^{2} \varphi}{\partial t^{2}}-\frac{1}{2}\left|\nabla \varphi^{\prime}\right|_{\varphi}^{2}\right) \operatorname{det} g=\epsilon \cdot \operatorname{det} g_{0}
$$

By definition,

$$
\frac{d J}{d t}=\int_{V} \frac{\partial \varphi}{\partial t}\left(g^{\alpha \bar{\beta}} \chi_{\alpha \bar{\beta}}\right)
$$


Then (denote $\sigma=g^{\alpha \bar{\beta}} \chi_{\alpha \bar{\beta}}$ in the following calculation):

$$
\begin{aligned}
& \frac{d^{2} J}{d t^{2}}=\int_{V}(\left.\frac{\partial^{2} \varphi}{\partial t^{2}} \sigma-\frac{\partial \varphi}{\partial t} g^{\alpha \bar{\beta}}\left(\frac{\partial \varphi}{\partial t}\right)_{, \bar{\beta} r} g^{r \bar{\delta}} \chi_{\alpha \bar{\delta}}+\frac{\partial \varphi}{\partial t} \sigma \triangle_{g} \frac{\partial \varphi}{\partial t}\right) \operatorname{det} g \\
&=\int_{V}\left(\frac{\partial^{2} \varphi}{\partial t^{2}} \sigma-\frac{\partial \varphi}{\partial t} g^{\alpha \bar{\beta}}\left(\frac{\partial \varphi}{\partial t}\right)_{, \bar{\beta} r} g^{r \bar{\delta}} \chi_{\alpha \bar{\delta}}-\left(\frac{\partial \varphi}{\partial t}\right)_{, r} \sigma g^{r \bar{\delta}}\left(\frac{\partial \varphi}{\partial t}\right)_{, \bar{\delta}}\right.\left.\quad-\frac{\partial \varphi}{\partial t} g^{\alpha \bar{\beta}} \chi_{\alpha \bar{\beta}, \bar{\delta}} g^{r \bar{\delta}}\left(\frac{\partial \varphi}{\partial t}\right)_{, r}\right) \operatorname{det} g \\
&=\int_{V}\left(\left(\frac{\partial^{2} \varphi}{\partial t^{2}}-\frac{1}{2}\left|\nabla \frac{\partial \varphi}{\partial t}\right|_{g}^{2}\right) \sigma-\frac{\partial \varphi}{\partial t} g^{\alpha \bar{\beta}}\left(\frac{\partial \varphi}{\partial t}\right)_{, \bar{\beta} r} g^{r \bar{\delta}} \chi_{\alpha \bar{\delta}}\right. \\
&\left.\quad-\frac{\partial \varphi}{\partial t}\left(g^{\alpha \bar{\beta}} \chi_{\alpha \bar{\delta}} g^{r \bar{\delta}}\right)_{, \bar{\beta}}\left(\frac{\partial \varphi}{\partial t}\right)_{, r}\right) \operatorname{det} g \\
&=\int_{V}\left(\left(\frac{\partial^{2} \varphi}{\partial t^{2}}-\frac{1}{2}\left|\nabla \frac{\partial \varphi}{\partial t}\right|_{g}^{2}\right)\left(g^{\alpha \bar{\beta}} \chi_{\alpha \bar{\beta}}\right)+\left(\frac{\partial \varphi}{\partial t}\right)_{, \bar{\beta}}\left(g^{\alpha \bar{\beta}} \chi_{\alpha \bar{\delta}} g^{r \bar{\delta}}\right)\left(\frac{\partial \varphi}{\partial t}\right)_{, r}\right) \operatorname{det} g \\
&=\int_{V}\left(\frac{\partial \varphi}{\partial t}\right)_{, \bar{\beta}}\left(g^{\alpha \bar{\beta}} \chi_{\alpha \bar{\delta}} g^{r \bar{\delta}}\right)\left(\frac{\partial \varphi}{\partial t}\right)_{, r} \operatorname{det} g \geq 0 .
\end{aligned}
$$

The last equality holds along any $C^{1,1}$ geodesic.

By Donaldson's viewpoint, we can view $J$ as a moment map, and we denote the energy of $J$ as $E$, then $E=\int_{V}\left(\operatorname{tr}_{g}(\chi)\right)^{2} \operatorname{det} g$.

Proposition 3.3. $E$ has same critical point as $J$. $E$ is decreasing by the gradient flow of $J$.

Proof. Let $\sigma=t r_{g} \chi$. All of the derivatives, norm and integration are taken with respect to $g$ in the following calculation:

$$
\begin{aligned}
\delta_{v} E(g) & =\int_{V}\left(2 \sigma\left(-\sum_{\alpha, \beta, r, \delta=1}^{n} g^{\alpha \bar{\beta}} v_{, \bar{\beta}} g^{r \bar{\delta}} \chi_{\alpha \bar{\delta}}\right)+\sigma^{2} \triangle_{g} v\right) \operatorname{det} g \\
& =\int_{V}\left(2 \sum_{\alpha, \beta, r, \delta=1}^{n}\left(g^{\alpha \bar{\beta}} \sigma_{, \bar{\beta}} v_{, r} g^{r \bar{\delta}} \chi_{\alpha \bar{\delta}}+g^{\alpha \bar{\beta}} \sigma v_{, r} g^{r \bar{\delta}} \chi_{\alpha \bar{\delta}, \bar{\beta}}\right)-2 \sigma \sigma_{, \bar{\delta}} v_{, r} g^{\bar{\delta} r}\right) \\
& =\int_{V}\left(2 \sum_{\alpha, \beta, r, \delta=1}^{n}\left(g^{\alpha \bar{\beta}} \sigma_{, \bar{\beta}} v_{, r} g^{r \bar{\delta}} \chi_{\alpha \bar{\delta}}+g^{\alpha \bar{\beta}} \sigma v_{, r} g^{r \bar{\delta}} \chi_{\alpha \bar{\beta}, \bar{\delta}}\right)-2 \sigma \sigma_{, \bar{\delta}} v_{, r} g^{\bar{\delta} r}\right) \\
& =2 \int_{V}\left(\sum_{\alpha, \beta, r, \delta=1}^{n} g^{\alpha \bar{\beta}} \sigma_{, \bar{\beta}} v_{, r} g^{r \bar{\delta}} \chi_{\alpha \bar{\delta}}\right)=-2 \int_{V} \sum_{\alpha, \beta, r, \delta=1}^{n}\left(g^{\alpha \bar{\beta}} \sigma_{, \bar{\beta}} \chi_{\alpha \bar{\delta}} g^{r \bar{\delta}}\right)_{, r} v .
\end{aligned}
$$


The Euler-Lagrange equation for functional $E$ is:

$$
\sum_{\alpha, \beta, r, \delta=1}^{n}\left(g^{\alpha \bar{\beta}} \sigma \overline{, \bar{\beta}} \chi_{\alpha \bar{\delta}} g^{r \bar{\delta}}\right)_{, r}=0
$$

The left hand side is a divergence form and the equation holds on the manifold without boundary. Taking the differentiation inside the brake, we have

$$
\sum_{\alpha, \beta, r, \delta=1}^{n}\left(g^{\alpha \bar{\beta}} \sigma_{, r \bar{\beta}} \chi_{\alpha \bar{\delta}} g^{r \bar{\delta}}\right)+\sum_{\alpha \beta=1}^{n} \sigma_{, \alpha} \sigma_{, \bar{\beta}} g^{\alpha \bar{\beta}}=0
$$

where we have used the identity $\chi_{\alpha \bar{\delta}, r} g^{r \bar{\delta}}=\left(\chi_{r \bar{\delta}} g^{r \bar{\delta}}\right)_{, \alpha}$ since $\chi$ is a closed $(1,1)$ form. At maximum or minimum point of $\sigma$, we have $\sigma_{, \alpha}=0$. Thus $\sigma_{, \alpha \bar{\beta}}=0$ at the critical point of $\sigma$ since $\chi$ is strictly positive $(1,1)$ form. Thus $\sigma=$ const identically. One can also prove this via integration by parts. Multiple $\sigma$ in both sides of equation (3.1) and integrating over the entire manifolds, we have

$$
\begin{aligned}
0= & \int_{V} \sum_{\alpha, \beta, r, \delta=1}^{n}\left(g^{\alpha \bar{\beta}} \sigma_{, r \bar{\beta}} \chi_{\alpha \bar{\delta}} g^{r \bar{\delta}}\right) \sigma+\sum_{\alpha \beta=1}^{n} \sigma_{, \alpha} \sigma_{, \bar{\beta}} g^{\alpha \bar{\beta}} \sigma \\
= & -\int_{V} \sum_{\alpha, \beta, r, \delta=1}^{n}\left(g^{\alpha \bar{\beta}} \sigma_{, r} \chi_{\alpha \bar{\delta}, \bar{\beta}} g^{r \bar{\delta}}\right) \sigma-\int_{V} \sum_{\alpha, \beta, r, \delta=1}^{n}\left(g^{\alpha \bar{\beta}} \sigma_{, r} \chi_{\alpha \bar{\delta}} g^{r \bar{\delta}}\right) \sigma_{\bar{\beta}} \\
& +\sum_{\alpha \beta=1}^{\alpha \beta} \sigma_{, \alpha} \sigma_{, \bar{\beta}} g^{\alpha \bar{\beta}} \sigma \\
= & -\int_{V} \sum_{\alpha, \beta, r, \delta=1}^{n}\left(g^{\alpha \bar{\beta}} \sigma_{, r} \chi_{\alpha \bar{\delta}} g^{r \bar{\delta}}\right) \sigma_{, \bar{\beta}} .
\end{aligned}
$$

Thus $\sigma=$ const in the manifold $V$ since $\chi$ is a strictly positive $(1,1)$ form.

Now, along a gradient flow of $J$, we have $\frac{\partial \varphi}{\partial t}=c-t r_{g} \chi=c-\sigma$. Thus,

$$
\begin{aligned}
\frac{d E}{d t} & =2 \int_{V}\left(\sum_{\alpha, \beta, r, \delta=1}^{n} g^{\alpha \bar{\beta}} \sigma_{, \bar{\beta}}(c-\sigma)_{, r} g^{r \bar{\delta}} \chi_{\alpha \bar{\delta}}\right) \\
& =-2 \int_{V}\left(\sum_{\alpha, \beta, r, \delta=1}^{n} g^{\alpha \bar{\beta}} \sigma_{, \bar{\beta}} \sigma_{, r} g^{r \bar{\delta}} \chi_{\alpha \bar{\delta}}\right) \leq 0 .
\end{aligned}
$$

The equality holds unless $\sigma$ is a constant. Thus $E$ is strictly decreasing under the gradient flow of $J$. 
Proposition 3.4. Any critical point of $E$ is a local minimizer.

Proof. Recall the first variation of $E$ is:

$$
\delta_{v} E(g)=2 \int_{V}\left(\sum_{\alpha, \beta, r, \delta=1}^{n} g^{\alpha \bar{\beta}} \sigma_{, \bar{\beta}} v_{, r} g^{r \bar{\delta}} \chi_{\alpha \bar{\delta}}\right) .
$$

Now we calculate the second variation of $E$ in $u, v$ direction, and substitute the condition for being critical point $\sigma=$ const, we have

$$
\begin{aligned}
\delta_{u} \delta_{v} E(g)= & -2 \int_{V}\left(\sum_{\alpha, \beta, r, \delta=1}^{n} g^{\alpha \bar{\beta}}\left(\sum_{i, j, k, l=1}^{n} g^{i \bar{j}} u_{, \bar{j} k} g^{k \bar{l}} \chi_{k \bar{l}}\right)_{, \bar{\beta}} v_{, r} g^{r \bar{\delta}} \chi_{\alpha \bar{\delta}}\right) \\
= & 2 \int_{V}\left(\sum_{\alpha, \beta, r, \delta, i, j, k, l=1}^{n} g^{\alpha \bar{\beta}} g^{i \bar{j}} u_{, \bar{j} k} g^{k \bar{l}} \chi_{k \bar{l}} v_{, r \bar{\beta}} g^{r \bar{\delta}} \chi_{\alpha \bar{\delta}}\right) \\
& +2 \int_{V}\left(\sum_{\alpha, \beta, r, \delta, i, j, k, l=1}^{n} g^{i \bar{j}} u_{, j k} g^{k \bar{l}} \chi_{k \bar{l}} v_{, r} g^{r \bar{\delta}} g^{\alpha \bar{\beta}} \chi_{\alpha \bar{\delta}, \bar{\beta}}\right) \\
= & \int_{V}\left(\sum_{\alpha, \beta, r, \delta, i, j, k, l=1}^{n} g^{\alpha \bar{\beta}} g^{i \bar{j}} u_{, \bar{j} k} g^{k \bar{l}} \chi_{k \bar{l}} v_{, r} \bar{\beta} g^{r \bar{\delta}} \chi_{\alpha \bar{\delta}}\right) \\
& +2 \int_{V}\left(\sum_{\alpha, \beta, r, \delta, i, j, k, l=1}^{n} g^{i \bar{j}} u_{, \bar{j} k} g^{k \bar{l}} \chi_{k \bar{l}} v_{, r} g^{r \bar{\delta}} \sigma_{, \bar{\delta}}\right) \\
= & \int_{V}\left(\sum_{\alpha, \beta, r, \delta, i, j, k, l=1}^{n} g^{\alpha \bar{\beta}} g^{i \bar{j}} u_{, \bar{j} k} g^{k \bar{l}} \chi_{k \bar{l}} v_{, r \bar{\beta}} g^{r \bar{\delta}} \chi_{\alpha \bar{\delta}}\right)>0 .
\end{aligned}
$$

The last equality holds since $\sigma=$ const at the critical point. Also, the last inequality holds as a symmetric form. $Q E D$.

Proposition 3.5. Under the gradient flow of $J$, the length of any smooth curve strictly decreases. Moreover, the distance between any two points decreases as well.

Proof. Suppose $\varphi(s):[0,1] \rightarrow \mathcal{H}$ is a smooth curve in the space of Kähler potentials. Now consider the energy of this curves as

$$
E n=\int_{s=0}^{1} \int_{V}\left(\frac{\partial \varphi}{\partial s}\right)^{2} \operatorname{det} g .
$$


Then, under gradient flow of $J$, suppose the energy becomes $E n(t)$. We want to show that the energy is strictly decreasing under this flow (All of the derivatives, norm and integration are taken with respect to $g+d s^{2}$ in the following calculation:).

$$
\begin{aligned}
\frac{d E n(t)}{d t}= & 2 \int_{s=0}^{1} \int_{V}\left(\frac{\partial \varphi}{\partial s} \frac{\partial^{2} \varphi}{\partial s \partial t}+\left(\frac{\partial \varphi}{\partial s}\right)^{2} \triangle_{g} \frac{\partial \varphi}{\partial t}\right) \operatorname{det} g \\
= & -2 \int_{s=0}^{1} \int_{V}\left(\frac{\partial \varphi}{\partial s} \frac{\partial \sigma}{\partial s}+\left(\frac{\partial \varphi}{\partial s}\right)^{2} \triangle_{g} \sigma\right) \operatorname{det} g \\
= & 2 \int_{s=0}^{1} \int_{V} \frac{\partial \varphi}{\partial s} \cdot\left(\sum_{\alpha, \beta, r, \delta=1}^{n} g^{\alpha \bar{\beta}}\left(\frac{\partial \varphi}{\partial s}\right)_{, \bar{\beta} r} g^{r \bar{\delta}} \chi_{\alpha \bar{\delta}}\right)+\frac{\partial \varphi}{\partial s}\left(\frac{\partial \varphi}{\partial s}\right)_{, \alpha} \sigma \overline{,}_{, \bar{\beta}} g^{\alpha \bar{\beta}} \\
=- & 2 \int_{s=0}^{1} \int_{V} \sum_{\alpha, \beta, r, \delta=1}^{n}\left(g^{\alpha \bar{\beta}}\left(\frac{\partial \varphi}{\partial s}\right)_{, \bar{\beta}} g^{\alpha \bar{\beta}}\left(\frac{\partial \varphi}{\partial s}\right)_{, r} g^{r \bar{\delta}} \chi_{\alpha \bar{\delta}}\right. \\
& \left.+\frac{\partial \varphi}{\partial s} \cdot g^{\alpha \bar{\beta}}\left(\frac{\partial \varphi}{\partial s}\right)_{, r} g^{r \bar{\delta}} \chi_{\alpha \bar{\delta}, \bar{\beta}}\right)^{n} \int_{s=0}^{1} \int_{V} \frac{\partial \varphi}{\partial s}\left(\frac{\partial \varphi}{\partial s}\right)_{, \alpha} \sigma_{, \bar{\beta}} g^{\alpha \bar{\beta}} \\
=- & \int_{s=0}^{1} \int_{V} \sum_{\alpha, \beta, r, \delta=1}^{n}\left(g^{\alpha \bar{\beta}}\left(\frac{\partial \varphi}{\partial s}\right)_{, \bar{\beta}} g^{\alpha \bar{\beta}}\left(\frac{\partial \varphi}{\partial s}\right)_{, r} g^{r \bar{\delta}} \chi_{\alpha \bar{\delta}}\right. \\
& \left.+\frac{\partial \varphi}{\partial s} \cdot\left(\frac{\partial \varphi}{\partial s}\right)_{, r} g^{r \bar{\delta}} \sigma_{, \bar{\delta}}\right)+2 \int_{s=0}^{1} \int_{V} \frac{\partial \varphi}{\partial s}\left(\frac{\partial \varphi}{\partial s}\right)_{, \alpha} \sigma{ }_{, \bar{\beta}} g^{\alpha \bar{\beta}} \\
= & -2 \int_{s=0}^{1} \int_{V} \sum_{\alpha, \beta, r, \delta=1}^{n}\left(g^{\alpha \bar{\beta}}\left(\frac{\partial \varphi}{\partial s}\right)_{, \bar{\beta}} g^{\alpha \bar{\beta}}\left(\frac{\partial \varphi}{\partial s}\right)_{, r} g^{r \bar{\delta}} \chi_{\alpha \bar{\delta}}\right) \leq 0 .
\end{aligned}
$$

The equality holds unless $\frac{\partial \varphi}{\partial s}$ is a constant in $V \times[0,1]$ or the geodesic is trivial.

If the $J$ flow exists for long time, then this will imply that $J$ flow decreases distance between any two points in $\mathcal{H}$. We will deal with the long term existence of the $J$ flow in the next section.

There are surprising similarities between this two group functions: the first group is K energy and Calabi energy, both are well known, but perhaps not well understood. The second group is our $J$ function and its norm $E$. Here $J$ plays the "role" of K energy and $E$ plays the role of Calabi energy. Calabi first proved in [3] that critical point of Calabi energy minimizes Calabi energy locally, one should compare this to proposition 3 aforementioned. In [4], we showed that Calabi flow (gradient flow of $\mathrm{K}$ energy) decreases 
the length of any smooth curves in the space of Kähler metrics, one should compare this to proposition 4 aforementioned. In [9], we prove that critical point for Calabi energy is unique if $C_{1}(V)<0$, and in general it is conjectured that critical point of Calabi energy is unique in each Kähler class, one should compare this to Proposition 1 aforementioned. The list of similarities could go on and on. The critical point for Calabi energy is well known. The critical point of $E$ is not known and is also not clear about its importance. But the similarity between $E$ and Calabi energy makes it clear: to study the critical point of $E$ or its gradient flow, is amount to study a junior version of extremal metrics or Calabi flow. The insight and technique we learned from the critical point of $J$ must be helpful to understand extremal metrics and Calabi flow.

\section{4. $C^{2}$ estimate of heat flow depending time $t$.}

Lemma 4.1. $\sigma>0$ is bounded from above and below along the gradient flow of $J$.

Proof. Taking second derivatives with respect time, we have

$$
\frac{\partial^{2} \varphi}{\partial t^{2}}=\sum_{\alpha, \beta, r, \delta=1}^{n} g^{\alpha \bar{\beta}}\left(\frac{\partial \varphi}{\partial t}\right)_{, \bar{\beta} r} g^{r \bar{\delta}} \chi_{\alpha \bar{\delta}}
$$

By ordinary maximum principle for parabolic equation, we have $\max _{V} \frac{\partial \varphi}{\partial t} \downarrow$ and $\min _{V} \frac{\partial \varphi}{\partial t} \uparrow$ as $t$ increases. Since $\frac{\partial \varphi}{\partial t}=c-\sigma$, we then prove this lemma.

Corollary 4.2. Under the gradient flow of $J$, metric is strictly bounded from below.

Theorem 4.3. There exists a const $C(t)$ depends on $t$ only, such that $\omega_{0}+$ $\partial \bar{\partial} \varphi \leq C \cdot \omega_{0}$.

Proof. Consider the heat flow equation:

$$
\frac{\partial \varphi}{\partial t}=c-\sum_{\alpha, \beta=1}^{n} g^{\alpha \bar{\beta}} \chi_{\alpha \bar{\beta}}
$$


We are now taking first and second derivatives on both side of this equation. In the following calculation, all indices are running from 1 to $n$. Whenever there is a repeated indices, that automatically means summation from 1 to $n$.

$$
\frac{\partial \varphi, i}{\partial t}=g^{\alpha \bar{r}} g_{\bar{r} \delta, i} g^{\delta \bar{\beta}} \chi_{\alpha \bar{\beta}}-g^{\alpha \bar{\beta}} \chi_{\alpha \bar{\beta}, i} .
$$

Now, let us take another derivative on both sides of the above equation, we have

$$
\begin{gathered}
\frac{\partial \varphi_{i \bar{j}}}{\partial t}=g^{\alpha \bar{r}} g_{\bar{r} \delta, \bar{j}} g^{\delta \bar{\beta}} \chi_{\alpha \bar{\beta}}-g^{\alpha \bar{a}} g_{\bar{a} b, \bar{j}} g^{b \bar{r}} g_{\bar{r} \delta, i} g^{\delta \bar{\beta}} \chi_{\alpha \bar{\beta}}-g^{\alpha \bar{r}} g_{\bar{r} \delta, i} g^{\delta \bar{a}} g_{\bar{a} b, \bar{j}} g^{b \bar{\beta}} \chi_{\alpha \bar{\beta}} \\
-g^{\alpha \bar{\beta}} \bar{j} \chi_{\alpha \bar{\beta}, i}-g^{\alpha \bar{\beta}} \chi_{\alpha \bar{\beta}, i \bar{j}} .
\end{gathered}
$$

Indices after comma represents partial derivatives in above two formulas. Now multiple the above equation with $\chi^{i \bar{j}}$ and summarized everything. Denote $F=\chi^{i \bar{j}} g_{i \bar{j}}=\chi^{i \bar{j}}\left(g_{0} \bar{j}+\varphi_{, i \bar{j}}\right)$. Consider all differentials as covariant differentials with respect to metric $\chi$. Denote the bisectional curvature of $\chi$ as $R(\chi)$. We then have

$$
\begin{aligned}
\frac{\partial F}{\partial t}= & g^{\alpha \bar{r}} g_{\bar{r} \delta, i \bar{j}} g^{\delta \bar{\beta}} \chi_{\alpha \bar{\beta}} \chi^{i \bar{j}}-2 g^{\alpha \bar{a}} g_{\bar{a} b, \bar{j}} g^{b \bar{r}} g_{\bar{r} \delta, i} g^{\delta \bar{\beta}} \chi_{\alpha \bar{\beta}} \chi^{i \bar{j}}+g^{\alpha \bar{\beta}} R(\chi)_{\alpha \bar{\beta} i \bar{j}} \chi^{i \bar{j}} \\
= & g^{\alpha \bar{r}} g_{\bar{r} i, \delta \bar{j}} g^{\delta \bar{\beta}} \chi_{\alpha \bar{\beta}} \chi^{i \bar{j}}-2 g^{\alpha \bar{a}} g_{\bar{a} b, \bar{j}} g^{b \bar{r}} g_{\bar{r} \delta, i} g^{\delta \bar{\beta}} \chi_{\alpha \bar{\beta}} \chi^{i \bar{j}}+g^{\alpha \bar{\beta}} R(\chi)_{\alpha \bar{\beta} i \bar{j}} \chi^{i \bar{j}} \\
= & g^{\alpha \bar{r}}\left(g_{\bar{r} i, \bar{j} \delta}+R(\chi)_{i \bar{p} \delta \bar{j}} g_{p \bar{r}}-R(\chi)_{p \bar{r} \delta \bar{j}} g_{i \bar{p}}\right) g^{\delta \bar{\beta}} \chi_{\alpha \bar{\beta}} \chi^{i \bar{j}} \\
& \quad-2 g^{\alpha \bar{a}} g_{\bar{a} b, \bar{j}} g^{b \bar{r}} g_{\bar{r} \delta, i} g^{\delta \bar{\beta}} \chi_{\alpha \bar{\beta}} \chi^{i \bar{j}}+g^{\alpha \bar{\beta}} R(\chi)_{\alpha \bar{\beta} i \bar{j}} \chi^{i \bar{j}} \\
= & g^{\alpha \bar{r}} g_{i \bar{j}, \bar{r} \delta} g^{\delta \bar{\beta}} \chi_{\alpha \bar{\beta}} \chi^{i \bar{j}}+g^{\alpha \bar{r}}\left(R(\chi)_{i \bar{p} \delta \bar{j}} g_{p \bar{r}}-R(\chi)_{p \bar{r} \delta \bar{j}} g_{i \bar{p}}\right) g^{\delta \bar{\beta}} \chi_{\alpha \bar{\beta}} \chi^{i \bar{j}} \\
& \quad-2 g^{\alpha \bar{a}} g_{\bar{a} b, \bar{j}} g^{b \bar{r}} g_{\bar{r} \delta, i} g^{\delta \bar{\beta}} \chi_{\alpha \bar{\beta}} \chi^{i \bar{j}}+g^{\alpha \bar{\beta}} R(\chi)_{\alpha \bar{\beta} i \bar{j}} \chi^{i \bar{j}} .
\end{aligned}
$$

Next, we can choose a coordinate such that $\chi_{i \bar{j}}=\delta_{i \bar{j}}$ and $g_{i \partial j}=\lambda_{i} \delta_{i \partial j}$. Note that at this point, we have

$$
\sigma=\sum_{\alpha=1}^{n} \frac{1}{\lambda_{\alpha}}, \quad \text { and } \quad F=\sum_{i=1}^{n} \lambda_{i} .
$$

Note that $\chi$ is a fixed metric, then there exists a constant $C$ such that the following inequality holds (via some straightforward calculation):

$$
\begin{aligned}
& g^{\alpha \bar{r}}\left(R(\chi)_{i \bar{p} \delta \bar{j}} g_{p \bar{r}}-R(\chi)_{p \bar{r} \delta \bar{j}} g_{i \bar{p}}\right) g^{\delta \bar{\beta}} \chi_{\alpha \bar{\beta}} \chi^{i \bar{j}} \\
& =\frac{1}{\lambda_{\alpha}}\left(R(\chi)_{i \bar{\alpha} \delta \bar{j}} \lambda_{\alpha}-R(\chi)_{i \bar{\alpha} \delta \bar{j}} \lambda_{i}\right) \frac{1}{\lambda_{\delta}} \delta_{i j} \leq C\left(\sigma+\sigma^{2} F\right) .
\end{aligned}
$$

Moreover, it is easy to check that

$$
\left|g^{\alpha \bar{\beta}} R(\chi)_{\alpha \bar{\beta} i \bar{j}} \chi^{i \bar{j}}\right| \leq c \sigma
$$


for some constant $c$. Thus, we have

$$
\frac{\partial F}{\partial t}=\tilde{\triangle} F-2 g^{\alpha \bar{a}} g_{\bar{a} b, \bar{j}} g^{b \bar{r}} g_{\bar{r} \delta, i} g^{\delta \bar{\beta}} \chi_{\alpha \bar{\beta}} \chi^{i \bar{j}}+C \cdot\left(\sigma+\sigma^{2} F\right)
$$

where $\tilde{\triangle} f=g^{\alpha \bar{r}} f_{, \bar{r} \delta} g^{\delta \bar{\beta}} \chi_{\alpha \bar{\beta}}$ for any smooth function $f$. From here, we quickly imply that $F$ is bounded from above since $\sigma$ is uniformly bounded from above.

Recall the estimate of equation (3.2), we have

$$
\begin{aligned}
\int_{0}^{\infty} \frac{d E}{d t} d t & =-2 \int_{0}^{\infty} \int_{V}\left(\sum_{\alpha, \beta, r, \delta=1}^{n} g^{\alpha \bar{\beta}} \sigma_{, \bar{\beta}} \sigma_{, r} g^{r \bar{\delta}} \chi_{\alpha \bar{\delta}}\right) \operatorname{det} g d t \\
& =E(\infty)-E(0) \geq C .
\end{aligned}
$$

In other words,

$$
\int_{0}^{\infty} \int_{V}\left(\sum_{\alpha, \beta, r, \delta=1}^{n} g^{\alpha \bar{\beta}} \sigma_{, \bar{\beta}} \sigma_{, r} g^{r \bar{\delta}} \chi_{\alpha \bar{\delta}}\right) \operatorname{det} g d t \leq C .
$$

There exists a subsequence of $t_{i} \rightarrow \infty$ such that

$$
\left.\int_{V}\left(\sum_{\alpha, \beta, r, \delta=1}^{n} g^{\alpha \bar{\beta}} \sigma_{, \bar{\beta}} \sigma_{, r} g^{r \bar{\delta}} \chi_{\alpha \bar{\delta}}\right) \operatorname{det} g\right|_{t=t_{i}} \rightarrow 0 .
$$

This last expression, shall suggests that $\left.\sigma\right|_{t=t_{i}} \rightarrow c$ in some sense for some constant $c$.

Next we return to prove the Theorem 1.1.

Proof. Following from the interior estimate by Evans and Krylov, we can imply $C^{2, \alpha}$ estimate for any finite time $t$. Then standard elliptic regularity theorem would imply that $g$ is $C^{\infty}$ at any finite time. Thus the flow exists for long time. Then proposition 4 implies that $J$ flow decreases distance between any two points in $\mathcal{H}$.

\section{Uniform $C^{2}$ estimate for heat flow for manifolds with semi-positive definite curvature tensors..}

In this section, we assume that the bisectional curvature of $\chi$ is non-negative, we want to show that there exists a uniform bound on the second derivatives of $\varphi$. 
Theorem 5.1. the bisectional curvature of $\chi$ is non-negative, then there exists a uniform bound on the second derivatives of $\varphi$.

Proof. Following the calculation in the previous section, we have (equation 4.1):

$$
\begin{gathered}
\frac{\partial \varphi_{, i \bar{j}}}{\partial t}=g^{\alpha \bar{r}} g_{\bar{r} \delta, i \bar{j}} g^{\delta \bar{\beta}} \chi_{\alpha \bar{\beta}}-g^{\alpha \bar{a}} g_{\bar{a} b, \bar{j}} g^{b \bar{r}} g_{\bar{r} \delta, i} g^{\delta \bar{\beta}} \chi_{\alpha \bar{\beta}}-g^{\alpha \bar{r}} g_{\bar{r} \delta, i} g^{\delta \bar{a}} g_{\bar{a} b, \bar{j}} g^{b \bar{\beta}} \chi_{\alpha \bar{\beta}} \\
-g^{\alpha{ }_{\bar{j}}} \chi_{\alpha \bar{\beta}, i}-g^{\alpha \bar{\beta}} \chi_{\alpha \bar{\beta}, \bar{j}} .
\end{gathered}
$$

Simplify this equation by adopting differentiation in terms of metric $\chi$, we have

$$
\frac{\partial \varphi_{, i \bar{j}}}{\partial t}=g^{\alpha \bar{r}} g_{\bar{r} \delta, i \bar{j}} g^{\delta \bar{\beta}} \chi_{\alpha \bar{\beta}}-g^{\alpha \bar{a}} g_{\bar{a} b, \bar{j}} g^{b \bar{r}} g_{\bar{r} \delta, i} g^{\delta \bar{\beta}} \chi_{\alpha \bar{\beta}}-g^{\alpha \bar{r}} g_{\bar{r} \delta, i} g^{\delta \bar{a}} g_{\bar{a} b, \bar{j}} g^{b \bar{\beta}} \chi_{\alpha \bar{\beta}}
$$

Define an auxiliary tensor $T_{i \bar{j}}$ as

$$
T_{i \bar{j}}=g_{i \bar{j}}-C_{0} \cdot \chi_{i \bar{j}}=g_{0} \overline{i j}+\varphi_{i \bar{j}}-C_{0} \cdot \chi_{i \bar{j}} .
$$

Choose $C_{0}$ big enough so that $T_{i \bar{j}}<0$ as a tensor at time $t=0$. Claim: $T<0$ is preserved under this heat flow. Let us first obtain the flow equation for $T$. From equation (5.1), we have

$$
\begin{aligned}
\frac{\partial T_{, i \bar{j}}}{\partial t}= & g^{\alpha \bar{r}} T_{\bar{r} \delta, i \bar{j}} g^{\delta \bar{\beta}} \chi_{\alpha \bar{\beta}}-g^{\alpha \bar{a}} g_{\bar{a} b, \bar{j}} g^{b \bar{r}} g_{\bar{r} \delta, i} g^{\delta \bar{\beta}} \chi_{\alpha \bar{\beta}}-g^{\alpha \bar{r}} g_{\bar{r} \delta, i} g^{\delta \bar{a}} g_{\bar{a} b, \bar{j}} g^{b \bar{\beta}} \chi_{\alpha \bar{\beta}} \\
= & g^{\alpha \bar{r}} T_{\bar{r} i, \delta \bar{j}} g^{\delta \bar{\beta}} \chi_{\alpha \bar{\beta}}-g^{\alpha \bar{a}} g_{\bar{a} b, \bar{j}} g^{b \bar{r}} g_{\bar{r} \delta, i} g^{\delta \bar{\beta}} \chi_{\alpha \bar{\beta}}-g^{\alpha \bar{r}} g_{\bar{r} \delta, i} g^{\delta \bar{a}} g_{\bar{a} b, \bar{j}} g^{b \bar{\beta}} \chi_{\alpha \bar{\beta}} \\
= & g^{\alpha \bar{r}}\left(T_{\bar{r} i, \bar{j} \delta}+R(\chi)_{i \bar{p} \delta \bar{j}} T_{p \bar{r}}-R(\chi)_{p \bar{r} \delta \bar{j}} T_{i \bar{p}}\right) g^{\delta \bar{\beta}} \chi_{\alpha \bar{\beta}} \\
= & \quad g^{\alpha \bar{a}} g_{\bar{a} b, \bar{j}} g^{b \bar{r}} g_{\bar{r} \delta, i} g^{\delta \bar{\beta}} \chi_{\alpha \bar{\beta}}-g^{\alpha \bar{r}} g_{\bar{r} \delta, i} g^{\delta \bar{a}} g_{\bar{a} b, \bar{j}} g^{b \bar{\beta}} \chi_{\alpha \bar{\beta}} \\
& \quad-g^{\alpha \bar{a}} g_{\bar{a} b, \bar{j} \delta} g^{b \bar{r}} g_{\bar{r} \delta, i} g^{\delta \bar{\beta}} \chi_{\alpha \bar{\beta}}-g^{\alpha \bar{r}} g_{\bar{r} \delta, i} g^{\delta \bar{a}} g_{\bar{a} b, \bar{j}} g^{b \bar{\beta}} \chi_{\alpha \bar{\beta}} .
\end{aligned}
$$

Now we want to apply Hamilton's maximal principal for tensors. Since $T_{i \bar{j}}<0$ at $t=0$, we may suppose that there is a first time $t=t_{0}>0$ and a point $O$, where $T$ has a degenerate direction. We might assume that this direction is $\xi(O)=\left(\xi^{1}, \xi^{2}, \cdots \xi^{n}\right)$; and parallel transport this vector along a small neighborhood of $O$ by metric $\chi$, so we have constant parallel vector field in a small neighborhood $\mathcal{O}$ of point $O$. By definition, we have

$$
T_{i j} \bar{\xi}^{i} \overline{\xi^{j}}<0, \quad \forall t<t_{0} .
$$

and at $t=t_{0}$ we have

$$
T_{i \bar{j}} \xi^{i} \overline{\xi^{j}}(O)=0, \quad \text { and } T_{i \bar{j}} \leq 0 \quad \text { in } \mathcal{O} .
$$


In particular, at $t=t_{0}$ and at point $O$, we have $T_{i j} \xi^{i}=T_{i j} \overline{\xi^{j}}=0$. Now plugging everything into the equation $(5.2)$, we have (in $\mathcal{O}$ )

$$
\begin{aligned}
& \frac{\partial\left(T_{i \bar{j}} \xi^{i} \overline{\xi^{j}}\right)}{\partial t}=g^{\alpha \bar{r}} T_{i \bar{j}, \bar{r} \delta} \xi^{i \overline{\xi^{j}}} g^{\delta \bar{\beta}} \chi_{\alpha \bar{\beta}}+g^{\alpha \bar{r}}\left(R(\chi)_{i \bar{p} \delta} T_{\bar{j}}-R(\chi)_{p \bar{r} \delta \bar{j}} T_{i \bar{p}}\right) g^{\delta \bar{\beta}} \chi_{\alpha \bar{\beta}} \xi^{i} \overline{\xi^{j}} \\
& -g^{\alpha \bar{a}} g_{\bar{a} b, \bar{j}} g^{b \bar{r}} g_{\bar{r} \delta, i} g^{\delta \bar{\beta}} \chi_{\alpha \bar{\beta}} \xi^{i} \overline{\xi^{j}}-g^{\alpha \bar{r}} g_{\bar{r} \delta, i} g^{\delta \bar{a}} g_{\bar{a} b, \bar{j}} g^{b \bar{\beta}} \chi_{\alpha \bar{\beta}} \xi^{i} \overline{\xi^{j}} \\
& =\tilde{\triangle}\left(T_{i \bar{j}} \xi^{i} \overline{\xi^{j}}\right)+g^{\alpha \bar{r}} R(\chi)_{i \bar{p} \delta \bar{j}} \xi^{i} \overline{\xi^{j}} T_{p \bar{r}} g^{\delta \bar{\beta}} \chi_{\alpha \bar{\beta}}-g^{\alpha \bar{r}} R(\chi)_{p \bar{r} \delta \bar{j}} T_{i \bar{p}} \xi^{i} g^{\delta \bar{\beta}} \chi_{\alpha \bar{\beta}} \overline{\xi^{j}} \\
& -g^{\alpha \bar{a}} g_{\bar{a} b, \bar{j}} g^{b \bar{r}} g_{\bar{r} \delta, i} g^{\delta \bar{\beta}} \chi_{\alpha \bar{\beta}} \xi^{i} \overline{\xi^{j}}-g^{\alpha \bar{r}} g_{\bar{r} \delta, i} g^{\delta \bar{a}} g_{\bar{a} b, \bar{j}} g^{b \bar{\beta}} \chi_{\alpha \bar{\beta}} \xi^{i} \overline{\xi^{j}} .
\end{aligned}
$$

Now at Point $O$ and at time $t=t_{0}$, by ordinary maximum principle, we have $\tilde{\triangle}\left(T_{i \bar{j}} \xi^{\xi^{j} \overline{\xi^{j}}}\right) \leq 0$. Moreover, $g^{\alpha \bar{r}} R(\chi)_{p \bar{r} \delta \bar{j}} T_{i \bar{p}} \xi^{i} g^{\delta \bar{\beta}} \chi_{\alpha \bar{\beta}} \overline{\xi^{j}}(O)=0$ and $g^{\alpha \bar{r}} R(\chi)_{i \bar{p} \delta \bar{j}} \xi^{i} \overline{\xi^{j}} T_{p \bar{r}} g^{\delta \bar{\beta}} \chi_{\alpha \bar{\beta}} \leq 0$ since $R(\chi)$ is a non-negative tensor while $T$ is a non-positive tensor. Thus

$$
\frac{\partial\left(T_{i j} \xi^{i} \overline{\xi^{j}}\right)}{\partial t}(O) \leq 0
$$

This implies that $T$ will remain non-positive. In other words,

$$
g_{i \bar{j}} \leq C_{0} \cdot \chi_{i \bar{j}}
$$

holds for all $t$ where the flow exists. Thus, all of the second derivatives of $\varphi$ is bounded from above.

Finally, we want to prove Theorem 1.2.

Proof. as before, following from the interior estimate by Evans and Krylov, we can obtian a uniform $C^{2, \alpha}$ estimate for any finite time $t$ from the theorem 3 above. Then standard elliptic regularity theorem would imply that $g$ is $C^{\infty}$ at any finite time. Thus the flow exists for long time. Since the $C^{2, \alpha}$ estimate is uniform (independent of time $t$ ), thus the flow converges to a critical point of $J$. at least by sequence. The uniqueness of sequential limit is provided by the fact that $J$ is strictly convex.

\section{References.}

[1] S. Bando. On the three dimensional compact Kähler manifolds of nonnegative bisectional curvature. J. D. G., 19:283-297, 1984.

[2] S. Bando and T. Mabuchi. Uniquness of Einstein Kähler metrics modulo connected group actions. In Algebraic Geometry, Advanced Studies in Pure Math., 1987. 
[3] E. Calabi. Extremal Kähler metrics. In Seminar on Differential Geometry, volume 16 of 102, pages 259-290. Ann. of Math. Studies, University Press, 1982.

[4] E. Calabi and X. X. Chen. Space of Kähler metrics (II). J. Diff. Geom., 61:173-193, 2002.

[5] H. D. Cao. Deformation of Kähler metrics to Kähler-Einstein metrics on compact Kähler manifolds. Invent. Math., 81:359-372, 1985.

[6] H. D. Cao. On Harnack's inequalities for the Kähler-Ricci flow. Invent. Math., 109:247-263, 1992.

[7] H. D. Cao and B. Chow. Recent developments on the ricci flow. Bull. Amer. Math. Soc, 36(1):59-74, 1999.

[8] X. X. Chen. On lower bound of the Mabuchi energy and its application. International Mathematics Research Notices, 12, 2000.

[9] X. X. Chen. Space of Kähler metrics. Journal of Differential Geometry,, $56: 189-234,2000$.

[10] B. Chow. The Ricci flow on the 2-sphere. J. Diff. Geom., 33:325-334, 1991.

[11] S.K. Donaldson. Moment maps and Diffeomorphisms. Asian J. Math., 3:1-15, 1999.

[12] S.K. Donaldson. Symmetric spaces, kahler geometry and Hamiltonian dynamics. Amer. Math. Soc. Transl. Ser. 2, 196, pages 13-33, 1999. Northern California Symplectic Geometry Seminar.

[13] J. Eells and J. Sampson. Harmonic mappings of Riemannian manifolds. Amer. J. Math., 86:109-160, 1964.

[14] R. Hamilton. Three-manifolds with positive Ricci curvature. J. Diff. Geom., 17:255-306, 1982.

[15] R. Hamilton. The Ricci flow on surfaces. Contemporary Mathematics, 71:237-261, 1988 .

[16] R. Hamilton. The formation of singularities in the Ricci flow, volume II. Internat. Press, 1993. 
[17] G. Huisken. Evolution of hypersurfaces by their curvature in riemannian manifolds. volume II of Proceedings of the International Congress of Mathematicians, Vol. II (Berlin, 1998).

[18] G. Huisken. Local and global behaviour of hypersurfaces moving by mean curvature. In Differential geometry: partial differential equations on manifolds, volume 54 of Proc. Sympos. Pure Math., 54, Part 1, Amer. Math. Soc.,, pages 175-191, 1990.

[19] T. Mabuchi. Some Sympletic geometry on compact kähler manifolds I. Osaka, J. Math., 24:227-252, 1987.

[20] N. Mok. The uniformization theorem for compact Kähler manifolds of non-negative holomorphic bisectional curvature. J. Differential Geom., $27: 179-214,1988$.

[21] S. Semmes. Complex monge-ampere and sympletic manifolds. Amer. J. Math., 114:495-550, 1992.

[22] W. X. Shi. Ricci deformation of the metric on complete non-compact riemannian manifolds. J. Differential Geom., 30:303-394, 1989.

[23] G. Tian. Some aspects of Kähler Geometry, 1997. Lecture note taken by Meike Akeveld. 